\title{
Nocturnal oxygen desaturation and spirometric parameters in adults with cystic fibrosis
}

\author{
M N Pond, S P Conway
}

\begin{abstract}
Background - Correction of nocturnal hypoxaemia in patients with cystic fibrosis may delay the development of pulmonary hypertension. Descriptive statistics used for nocturnal arterial oxygen saturation $\left(\mathrm{SpO}_{2}\right)$ lack uniformity. The relationship between $\mathrm{SpO}_{2}$ and spirometric parameters has not previously been explored in a large number of exacerbations in adult patients with cystic fibrosis.
\end{abstract}

Methods - Over a 21 month period overnight $\mathrm{Spo}_{2}$, forced expiratory volume in one second $\left(F E V_{1}\right)$, and forced vital capacity (FVC) were recorded on admission and discharge in 120 treatments of pulmonary exacerbations in 47 patients with cystic fibrosis who did not receive supplemental oxygen during recording. Nocturnal $\mathrm{SpO}_{2}$ was related to spirometric parameters for the whole group and individually in 11 patients, each of whom had at least five treatments.

Results - There was a close linear relationship between the percentage of the recording spent with $\mathrm{SpO}_{2}<90 \%$ and mean overnight $\mathrm{SpO}_{2}$. Mean $\mathrm{SpO}_{2}$ correlated moderately with percentage predicted $\mathrm{FEV}_{1}\left(\% \mathrm{FEV}_{1}\right), r=0 \cdot 6$, and poorly with percentage predicted FVC (\% FVC), $r=0 \cdot 34$. The relationship between mean $\mathrm{Spo}_{2}$ and \% $\mathrm{FEV}_{1}$ was non-linear at mean $\mathrm{Spo}_{2}$ $<89 \%$, but approximated to linearity above this value. After exclusion of treatments with mean $\mathrm{SpO}_{2}<89 \%$ the regression relationship between mean $\mathrm{SpO}_{2}$ and \% FEV was the same on admission and discharge. Individual correlation coefficients of mean $\mathrm{SpO}_{2}$ versus \% $\mathrm{FEV}_{1}$ in the 11 patients with repeated treatments ranged from 0.57 to 0.77 . The slopes of the regression lines did not differ, with a pooled slope of $0 \cdot 116$, but the intercepts varied widely.

Conclusions - In patients with cystic fibrosis mean overnight $\mathrm{SpO}_{2}$ can be substituted for percentage of recording $<90 \%$. The relationship between mean $\mathrm{SpO}_{2}$ and percentage predicted $\mathrm{FEV}_{1}$ is non-linear at low values of $\mathrm{SpO}_{2}$ and is not influenced by treatment of pulmonary exacerbations. Patients with cystic fibrosis desaturate at a uniform rate compared with percentage predicted $F E V_{1}$, but the value of $F E V_{1}$ at which desaturation first occurs varies between patients. The spirometric values do not accurately predict nocturnal desaturation in a cystic fibrosis population, but $\mathrm{FEV}_{1}$ is a useful guide in individual patients with moderate desaturation.

(Thorax 1995;50:539-542)

Keywords: cystic fibrosis, nocturnal oxygen desaturation, spirometry.

The value of oxygen therapy in cystic fibrosis is uncertain. In acute pulmonary exacerbations the mainstays of treatment are intravenous antibiotics, physiotherapy, and increased calorie intake. There are no published studies concerning the effects of oxygen therapy in this setting. Nocturnal hypoxaemia is common in cystic fibrosis s $^{1-6}$ and is likely to precede daytime hypoxaemia. ${ }^{467}$ The mechanism is probably a combination of hypoventilation and reduced functional residual capacity. ${ }^{38}$ Pulmonary artery pressure (PAP) is inversely correlated with $\mathrm{SpO}_{2}$ in cystic fibrosis, and decreases with supplemental oxygen. ${ }^{19}$ The development of pulmonary hypertension might be delayed by correction of nocturnal hypoxaemia.

A variety of descriptive statistics has been used for nocturnal $\mathrm{SpO}_{2}$ in cystic fibrosis - for example, $10 \%$ of an overnight recording with $\mathrm{Spo}_{2}<90 \%$ is usually taken to represent significant desaturation. ${ }^{4-610}$ The first aim of our study was to determine whether simple mean $\mathrm{SpO}_{2}$ or lowest recorded $\mathrm{Spo}_{2}$ could substitute for time spent with $\mathrm{SpO}_{2}<90 \%$. It is unclear from previous work whether nocturnal desaturation can be predicted by $\mathrm{FEV}_{1} .{ }^{5711}$ The second aim was to clarify the relationship between routinely measured spirometric parameters and nocturnal desaturation in a large number of acute pulmonary exacerbations in a cystic fibrosis population, and in a smaller number of individual patients with repeated admissions for treatments of exacerbations.

\section{Methods}

From February 1992 to November 1993 all patients with cystic fibrosis admitted for intravenous antibiotic treatment of pulmonary exacerbations underwent overnight $\mathrm{SpO}_{2}$ recording on the night of admission and on the night prior to discharge. The second recording was performed only after the decision to discharge had been taken. The decision to treat with intravenous antibiotics was made on the basis of an increase in respiratory symptoms and/or a decline in $\mathrm{FEV}_{1}$ or FVC of $10 \%$ or more compared with the patient's previous values. All patients were treated with two intravenous antipseudomonal antibiotics, 
Table 1 Admission and discharge values of analysed exacerbations (120 exacerbations in 47 patients)

\begin{tabular}{|c|c|c|c|}
\hline & Admission & Discharge & $\begin{array}{l}\text { ANOVA } \\
\text { (admission v discharge) }\end{array}$ \\
\hline $\begin{array}{l}\text { Mean (SD) \% predicted } \mathrm{FEV}_{1} \\
\text { Mean (SD) \% predicted FVC } \\
\text { Mean (SD) mean } \mathrm{SpO}_{2}(\%) \\
\text { Mean (SD) lowest } \mathrm{Spo}_{2}(\%) \\
\text { Median (IQ range) percentage recording with } \mathrm{Spo}_{2}<90 \% \\
\text { Median (IQ range) recording duration (hours) }\end{array}$ & $\begin{array}{ll}49 \cdot 9 & (21 \cdot 0) \\
76 \cdot 8 & (22 \cdot 6) \\
91 \cdot 86 & (3.59) \\
83 \cdot 17 & (7 \cdot 67) \\
3 & (0-20 \cdot 5) \\
7 \cdot 37 & (6 \cdot 7-7 \cdot 9)\end{array}$ & $\begin{array}{ll}62 \cdot 4 & (24 \cdot 0) \\
95 \cdot 7 & (23 \cdot 9) \\
93 \cdot 77 & (2 \cdot 46) \\
85 \cdot 71 & (5 \cdot 62) \\
0 & (0-2 \cdot 5) \\
7 \cdot 54 & (6 \cdot 7-7 \cdot 9)\end{array}$ & $\begin{array}{l}\mathrm{p}<0.0001 \\
\mathrm{p}<0.0001 \\
\mathrm{p}<0.0001 \\
\mathrm{p}=0.005 \\
\mathrm{p}<0.0001 \\
\mathrm{p}=\mathrm{NS}\end{array}$ \\
\hline
\end{tabular}

based on their most recent sputum pseudomonal sensitivities. Oxygen therapy was continued in those considered to be oxygendependent, but only newly commenced after demonstrating that $>10 \%$ of the admission $\mathrm{SpO}_{2}$ recording was below $90 \%$.

$\mathrm{SpO}_{2}$ was recorded using four Biox 3740 and one Biox 3700 oximeters (Ohmeda), with a

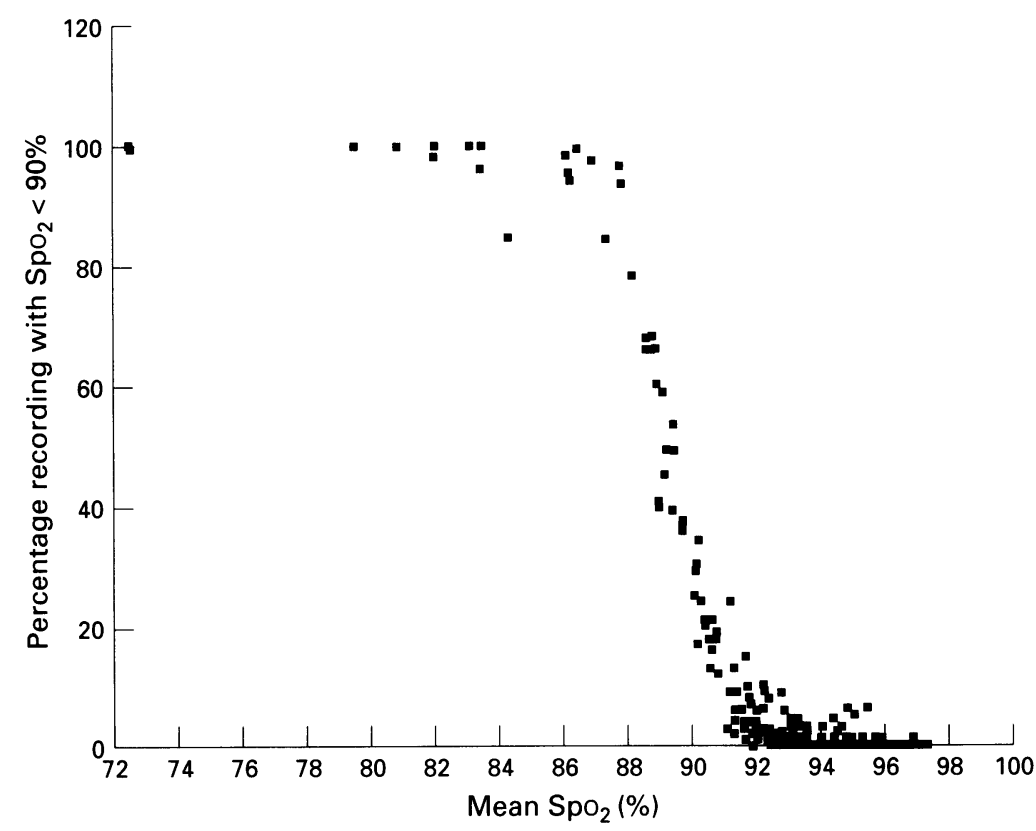

Figure 1 Percentage recording with $\mathrm{SpO}_{2}<90 \%$ versus mean in all recordings $(n=240)$.

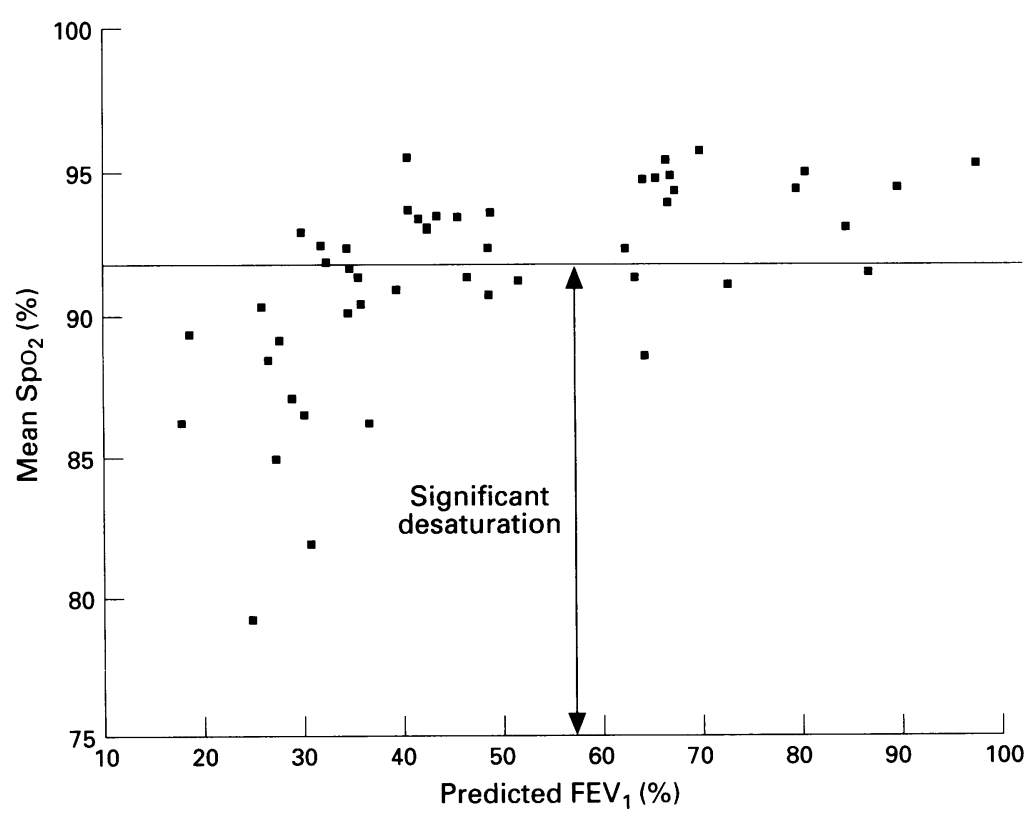

Figure 2 Mean $\mathrm{SpO}_{2}$ versus percentage predicted $\mathrm{FEV} V_{1}$. Each point represents mean of admission values in individual patients $(n=47)$. Significant desaturation $=$ mean $\mathrm{SpO}_{2}$ $<92 \%$. probe carefully attached to one finger. These oximeters have previously been validated. ${ }^{12}$ The presence of finger clubbing does not affect pulse oximetry results. ${ }^{13} \mathrm{SpO}_{2}$ was monitored only for the period when the patients were asleep. Where the duration of sleep exceeded eight hours only the last eight hours of the recording were analysed. On the day after recording oximeter data were analysed using inhouse software which automatically excluded artefactual desaturation. Each oximeter recording was also examined by an experienced observer (MNP) on a compressed time base to confirm the exclusion of artefact and to ensure that only the data pertaining to the previous night's recording were included for analysis (in cases with a record duration of less than eight hours). The following summary statistics were then calculated by the software: mean $\mathrm{SpO}_{2}$, lowest $\mathrm{SpO}_{2}$, duration of record, and time spent with $\mathrm{SpO}_{2}<90 \%$.

On the day of admission and the day of discharge $\mathrm{FEV}_{1}$ and $\mathrm{FVC}$ were recorded using one Vitalograph Compact spirometer. Patients were asked to repeat forced manoeuvres until the highest two readings differed by no more than $5 \% . \mathrm{FEV}_{1}$ and FVC obtained from the expiratory effort with the greatest $\mathrm{FEV}_{1}$ were then recorded and expressed as percentage predicted based on the patient's sex, age, and height.

Data were included for analysis only from those exacerbations which met the following 음 criteria: treatment solely in hospital (no element of home therapy); duration of treatment at least 10 days; and no supplemental oxygen $N$ used during either admission or discharge recordings.

\section{STATISTICAL ANALYSIS}

Because we aimed to identify patients with repeated qualifying exacerbations, variable 0 numbers of exacerbations were included for $\bar{O}$ each patient. Consequently, in the group analysis ANOVA was performed with patients as $\stackrel{\mathbb{Q}}{\varrho}$ a nested factor to determine improvement with treatment, mean values were calculated for each patient for correlation and regression, and regression analyses (including general linear modelling) were then weighted for number of exacerbations. Statistical calculations were performed with the Minitab programme.

\section{Results}

One hundred and twenty exacerbations in 47 patients fulfilled the criteria for inclusion in the analysis. Details of the exacerbations analysed 
Table 2 Predicted FEV $V_{1}$ and FVC and correlation of \% predicted FEV with mean $\mathrm{SpO}_{2}$ in 11 individuals with $\geqslant 5$ repeated exacerbations. Both admission and discharge values are included yielding a minimum of 10 observations per patient

\begin{tabular}{|c|c|c|c|c|c|}
\hline Patient no. & $n$ & $\begin{array}{l}\text { Mean \% } \\
\text { predicted } \\
F E V_{1}\end{array}$ & $\begin{array}{l}\text { Mean \% } \\
\text { predicted } \\
F V C\end{array}$ & $\begin{array}{l}\text { Mean } \\
\left(\text { mean } \mathrm{SpO}_{2}\right)\end{array}$ & $\begin{array}{l}\text { Correlation } \\
\text { coefficient }\end{array}$ \\
\hline $\begin{array}{r}1 \\
2 \\
3 \\
4 \\
5 \\
6 \\
7 \\
8 \\
9 \\
10 \\
11\end{array}$ & $\begin{array}{l}14 \\
10 \\
10 \\
10 \\
12 \\
10 \\
10 \\
12 \\
12 \\
18 \\
10\end{array}$ & $\begin{array}{l}70 \cdot 2 \\
33 \cdot 4 \\
40 \cdot 0 \\
42 \cdot 4 \\
54 \cdot 1 \\
95 \cdot 6 \\
86 \cdot 7 \\
40 \cdot 9 \\
36 \cdot 2 \\
44 \cdot 9 \\
65 \cdot 7\end{array}$ & $\begin{array}{r}106.4 \\
73.8 \\
56.8 \\
63.7 \\
73.2 \\
14.5 \\
116.9 \\
94.9 \\
82.4 \\
85.9 \\
93.1\end{array}$ & $\begin{array}{l}92 \cdot 52 \\
90 \cdot 43 \\
92 \cdot 93 \\
92 \cdot 85 \\
92 \cdot 35 \\
95 \cdot 36 \\
95 \cdot 77 \\
90 \cdot 58 \\
93 \cdot 20 \\
92 \cdot 30 \\
94 \cdot 25\end{array}$ & $\begin{array}{l}0.61 \\
0.65 \\
0.64 \\
0.63 \\
0.77 \\
0.73 \\
0.68 \\
0.77 \\
0.57 \\
0.72 \\
0.69\end{array}$ \\
\hline
\end{tabular}

are shown in table 1. As expected, significant improvements were seen in all the measured parameters.

The percentage of recording time with $\mathrm{Spo}_{2}$ $<90 \%$ is plotted against the mean $\mathrm{SpO}_{2}$ in fig 1 for admission and discharge values from all exacerbations. Between the limits of $0 \%$ and $100 \%$ the percentage of recording $<90 \%$ decreases linearly with increasing mean $\mathrm{Spo}_{2}$. There was a much weaker relation between the percentage of recording $<90 \%$ and lowest recorded $\mathrm{Spo}_{2}$. Significant hypoxaemia is usually considered to occur when $\mathrm{SpO}_{2}$ is $<90 \%$ for more than $10 \%$ of the recording. Mean $\mathrm{Spo}_{2}$ of $92 \%$ is $98 \%$ sensitive and $74 \%$ specific in predicting this value, with positive and negative predictive values of $74 \%$ and $99.5 \%$, respectively. The percentage of recording time with $\mathrm{SpO}_{2}<90 \%$ can therefore be substituted by the much simpler mean $\mathrm{Spo}_{2}$, with values of mean $\mathrm{SpO}_{2}<92 \%$ representing significant hypoxaemia.

Mean $\mathrm{Spo}_{2}$ is plotted against \% $\mathrm{FEV}_{1}$ on admission in fig 2 . The discharge scatterplot was similar, with the points shifted to the right (after improvement with treatment). There was a better correlation between mean $\mathrm{Spo}_{2}$ and \% $\mathrm{FEV}_{1} \quad(r=0.59$ on admission, 0.52 on discharge) than between mean $\mathrm{Spo}_{2}$ and \% FVC ( $r=0.38$ on admission, 0.35 on discharge).

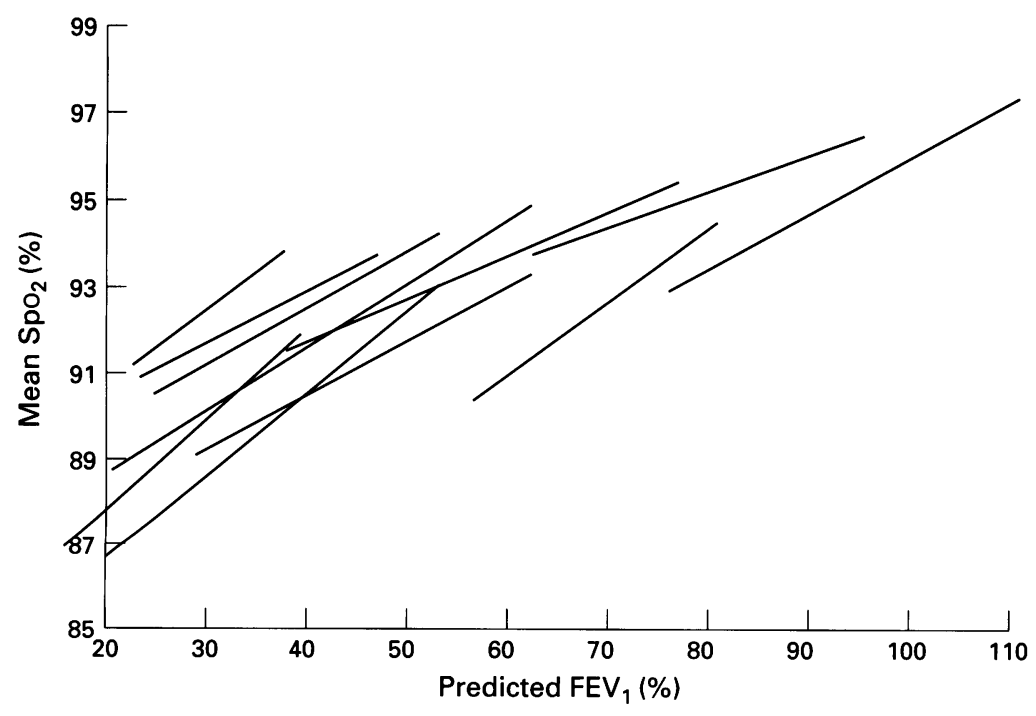

Figure 3 Regression of mean $\mathrm{SpO}_{2}$ on percentage predicted $\mathrm{FEV} V_{1}$. Each regression line represents one of 11 individual patients with five or more exacerbations. Admission and discharge values are included, yielding a minimum of 10 observations per patient. General linear model for difference between slopes $=N S$, for difference between intercepts $p<0 \cdot 005$. Pooled slope of mean $\mathrm{SpO}_{2}$ on percentage predicted $F E V_{1}=0 \cdot 116$.
The relation between mean $\mathrm{Spo}_{2}$ and $\% \mathrm{FEV}_{1}$ appeared to be linear until very low mean $\mathrm{Spo}_{2}$ values were recorded. Regression analysis with the exploratory lack of fit model confirmed that this was the case. For both the admission and discharge plots exclusion of patients with mean $\left(\right.$ mean $\left.\mathrm{SpO}_{2}\right)<89 \%(\mathrm{n}=8)$ resulted in a regression relationship that did not significantly differ from linearity. This resulted in a greater correlation coefficient of 0.61 on discharge, but the admission value was unchanged. Both regression equations were statistically significant and not significantly different from each other ( $p=N S$ using the general linear model):

mean $\mathrm{Spo}_{2}($ admission $)=90 \cdot 3+0.0471 \%$
$\mathrm{FEV}_{1}($ admission $)(\mathrm{p}<0.0001)$
mean $\mathrm{Spo}_{2}($ discharge $)=91 \cdot 6+0.0377 \%$ $\mathrm{FEV}_{1}$ (discharge) $(\mathrm{p}<0.0001)$

The relationship between mean $\mathrm{Spo}_{2}$ and $\mathrm{FEV}_{1}$ was explored further for the individual case in 11 patients who had had at least five eligible exacerbations during the period of the study. Since the same relationship between mean $\mathrm{Spo}_{2}$ and \% $\mathrm{FEV}_{1}$ holds on both admission and discharge, both of these data points were included, yielding a minimum of $10 \mathrm{ob}-$ servations per patient. Individual correlations between mean $\mathrm{SpO}_{2}$ and percentage predicted $\mathrm{FEV}_{1}$ are shown for these patients with the clinical characteristics in table 2 . The regression lines of these 11 patients are shown in fig 3. General linear modelling showed the differences between the slopes of the individual lines to be non-significant, and hence parallel, but the intercepts were significantly different $(\mathrm{p}<0.005)$. The pooled slope was $0 \cdot 116$.

\section{Discussion}

$\mathrm{SpO}_{2}$ during sleep remains at a steady level in patients with cystic fibrosis, with slightly lower values recorded during periods of REM sleep. ${ }^{8}$ Because of this lack of variation from baseline there is a close linear relationship between mean $\mathrm{SpO}_{2}$ and the percentage of recording time with $\mathrm{Spo}_{2}<90 \%$ (Pearson $r=-0.95$, fig 1). Mean $\mathrm{SpO}_{2}$ can therefore substitute for the cumbersome percentage of recording with $\mathrm{Spo}_{2}$ $<90 \%$ as a descriptive statistic. It is generally accepted that significant desaturation occurs when $\mathrm{SpO}_{2}$ is $<90 \%$ (corresponding to a $\mathrm{Po}_{2}$ of $8 \mathrm{kPa}$ at $\mathrm{pH} 7 \cdot 4$ ) for more than $10 \%$ of the overnight recording. ${ }^{4-610}$ This corresponds to a mean $\mathrm{Spo}_{2}$ of $92 \%$.

We observed a closer relationship between mean $\mathrm{Spo}_{2}$ and $\mathrm{FEV}_{1}$ than between mean $\mathrm{Spo}_{2}$ and FVC. Further analysis of our data was confined to mean $\mathrm{Spo}_{2}$ and $\mathrm{FEV}_{1}$. Exploratory regression of mean $\mathrm{SpO}_{2}$ on $\% \mathrm{FEV}_{1}$ revealed definite curvature at low values of $\mathrm{Spo}_{2}$ for both the admission and discharge cases (fig 2) which has not previously been described. This is not due to inaccuracy of the oximeters since they tend to overread in this range ${ }^{4}$ and the true relationship is therefore likely to be even more curvilinear. Given that the oxygen dis- 
sociation curve is sigmoid, it is likely that this relationship is non-linear over the whole range of $\mathrm{SpO}_{2}$, but for both the admission and discharge cases exclusion of patients with admission mean $\mathrm{SpO}_{2}<89 \%(\mathrm{n}=8)$ resulted in a regression that did not differ significantly from linearity. This allowed comparison of the admission and discharge plots with simple linear statistics. The relationship between mean $\mathrm{Spo}_{2}$ and $\mathrm{FEV}_{1}$ might be different on admission and discharge as the various therapeutic interventions employed in treating pulmonary exacerbations might have differential effects on $\mathrm{FEV}_{1}$ and nocturnal $\mathrm{SpO}_{2}$, and some subjects may require a night to acclimatise to hospital surroundings. ${ }^{14}$ However, both regression equations were very similar and not significantly different. This result may not apply to the very low mean $\mathrm{Spo}_{2}$ values that had to be excluded to allow the comparison. When patients are in a stable phase routinely measured clinical parameters usually fall between the admission and discharge values. The relationship between mean $\mathrm{Spo}_{2}$ and $\mathrm{FEV}_{1}$ is thus the same at the extremes of the variation in short term pulmonary function and it is likely that this relationship also applies when patients are in a stable phase.

It has been suggested that clinically significant desaturation does not occur in cystic fibrosis patients with predicted $\mathrm{FEV}_{1}>65 \%$. $^{5}$ In our study significant desaturation occurred in three patients ( $12 \%$ of the total who desaturated) with predicted $\mathrm{FEV}_{1}>65 \%$. It is probably not possible to identify a value of $\mathrm{FEV}_{1}$ above which desaturation will not occur in a cystic fibrosis population. Correlation between mean $\mathrm{Spo}_{2}$ and \% $\mathrm{FEV}_{1}$ for the group as a whole was modest, as found by previous authors, ${ }^{51011}$ but in the 11 patients with more than five treatments stronger individual correlations were found. All 11 patients desaturated at a similar rate relative to $\% \mathrm{FEV}_{1}$ (fig 3) with a pooled slope of $0 \cdot 116$ - that is, for each $10 \%$ decrease in $\% \mathrm{FEV}_{1}$ mean $\mathrm{Spo}_{2}$ decreased $1 \cdot 16 \%$. However, the value of $\%$ $\mathrm{FEV}_{1}$ at which desaturation first occurred varied widely between patients.

Spirometric parameters do not predict nocturnal desaturation during pulmonary ex- acerbations in a cystic fibrosis population, and desaturation must initially be specifically sought with overnight oximetry. However, since individual patients desaturate predictably in relation to $\mathrm{FEV}_{1}$, the $\mathrm{FEV}_{1}$ at which desaturation first occurs can subsequently serve as a marker of the presence of desaturation in that individual, reducing the need for subsequent overnight recordings during exacerbations. In the outpatient setting $\mathrm{FEV}_{1}$ can then guide whether pulmonary exacerbations are treated at home or in hospital where nocturnal desaturation would be more easily corrected, and $\mathrm{FEV}_{1}$ can also guide the timing of assessment of suitability for continuous home overnight oxygen.

1 Davidson A, Bossuyt A, Dab I. Acute effects of oxygen, nifedipine, and diltiazem in patients with cystic fibrosis and mild pulmonary hypertension. Pediatr Pulmonol 1989, 6:53-9.

2 Stokes DC, McBride JT, Wall MA, Erba G, Streider DJ. Sleep hypoxaemia in young adults with cystic fibrosis. $\mathrm{Am}$

f Dis Child 1980;134:741-3.
3 Francis PW, Muller NL, Gurwitz D, Milligan DWA, Levinson $\mathrm{H}$, Bryan AC. The hemoglobin desaturation - its occurrence during sleep in patients with cystic fibrosis. Am $\mathcal{f}$ Dis Child 1980;134:734-40.

4 Braggion C, Pradal U, Mastella G. Hemoglobin desaturation during sleep and daytime in patients with cystic $\vec{G}$ fibrosis and severe airway obstruction. Acta Paediatr 1992; 81:1002-6.

5 Versteegh FGA, Neijens HJ, Bogaard JM, Stam H, Robijn RJ, Kerrebijn KF. Relationship between pulmonary function, $\mathrm{O}_{2}$ saturation during sleep and exercise, and exercise responses in children with cystic fibrosis. Adv Cardiol 1986;35:151-5.

6 Versteegh FGA, Bogaard J, Neijens HJ, Kerrebijn KF. Relationship between airway obstruction, desaturation during exercise and nocturnal hypoxaemia in cystic fibrosis ing exercise and nocturnal hypoxaem

7 Coffey MJ, Fitzgerald MX, McNicholas WT. Comparison of oxygen desaturation during sleep and exercise in patients with cystic fibrosis. Chest 1991;100:659-62.

8 Muller NL, Francis PW, Gurwitz D, Levison H, Bryan AC. Mechanism of hemoglobin desaturation during rapid eye movement sleep in normal subjects and in patients with cystic fibrosis. Am Rev Respir Dis 1980;121:463-9.

9 Goldring RM, Fishman AP, Turino GM, Cohen HI, Denning CR, Andersen DH. Pulmonary hypertension and cor pulmonale in cystic fibrosis of the pancreas. f Pediatr 1964;65:501-24.

10 Smith DL, Freeman W, Cayton RM, Stableforth DE. Noc- oิ turnal hypoxaemia in cystic fibrosis: relationship to pulmonary function tests. Respir Med 1994;88:537-9.

11 Montgomery M, Wiebicke W, Bibi H, Pagtakhan RD, 을 Pasterkamp $\mathrm{H}$. Home measurement of oxygen saturation during sleep in patients with cystic fibrosis. Pediatr Pulmonol 1989;7:29-34.

12 Warley ARH, Mitchell JH, Stradling JR. Evaluation of the Ohmeda 3700 pulse oximeter. Thorax 1987;42:892-6.

13 Schoni MH, Hurliman R, Bruderer K. Pulse oximetry, digital clubbing and cystic fibrosis. Lancet 1987; 1 : 1317 .

14 Agnew HW, Webb WB, Williams RL. The first night effect: an EEG study of sleep. Psychopharmacology 1966;2:263-6. 